\title{
Prenatal dexamethasone exposure induces changes in nonhuman primate offspring cardiometabolic and hypothalamic- pituitary-adrenal axis function
}

\author{
Annick de Vries, ${ }^{1}$ Megan C. Holmes, ${ }^{1}$ Areke Heijnis, ${ }^{1}$ Jürgen V. Seier, ${ }^{2}$ Joritha Heerden, ${ }^{2}$ \\ Johan Louw, ${ }^{2}$ Sonia Wolfe-Coote, ${ }^{2}$ Michael J. Meaney, ${ }^{3}$ Naomi S. Levitt, ${ }^{4}$ and Jonathan R. Seckl ${ }^{1}$ \\ ${ }^{1}$ Endocrinology Unit, University of Edinburgh, The Queen's Medical Research Institute, Edinburgh, United Kingdom. 2Primate Unit, \\ Diabetes Research Group, South African Medical Research Council, Cape Town, South Africa. ${ }^{3}$ Douglas Hospital Research Center, McGill University, Montreal, \\ Quebec, Canada. ${ }^{4}$ Endocrine and Diabetes Unit, Department of Medicine, Faculty of Health Sciences, University of Cape Town, Cape Town, South Africa.
}

\begin{abstract}
Prenatal stress or glucocorticoid administration has persisting "programming" effects on offspring in rodents and other model species. Multiple doses of glucocorticoids are in widespread use in obstetric practice. To examine the clinical relevance of glucocorticoid programming, we gave 50,120 , or $200 \mu \mathrm{g} / \mathrm{kg} / \mathrm{d}$ of dexamethasone (dex50, dex120, or dex200) orally from mid-term to a singleton-bearing nonhuman primate, Chlorocebus aethiops (African vervet). Dexamethasone dose-dependently reduced maternal cortisol levels without effecting maternal blood pressure, glucose, electrolytes, or weight gain. Birth weight was unaffected by any dexamethasone dose, although postnatal growth was attenuated after dex120 and dex200. At 8 months of age, dex120 and dex200 offspring showed impaired glucose tolerance and hyperinsulinemia, with reduced (approximately $25 \%)$ pancreatic $\beta$ cell number at 12 months. Dex120 and dex200 offspring had increased systolic and diastolic blood pressures at 12 months. Mild stress produced an exaggerated cortisol response in dex200 offspring, implying hypothalamic-pituitary-adrenal axis programming. The data are compatible with the extrapolation of the glucocorticoid programming hypothesis to primates and indicate that repeated glucocorticoid therapy and perhaps chronic stress in humans may have long-term effects.
\end{abstract}

\section{Introduction}

Environmental signals during prenatal development often produce structural and functional effects that persist for the lifespan. Such effects are commonly dubbed fetal "programming." Studies of fetal programming could potentially explain human epidemiological findings indicating that low birth weight and other markers of an adverse intrauterine environment associate with a substantially greater incidence of cardiovascular, metabolic, and neuroendocrine disorders in later life (1-5).

We originally hypothesized that antenatal exposure to excess glucocorticoids might underpin the links between low birth weight and subsequent adult pathophysiology (6). We and others demonstrated that treatment of pregnant rats with the synthetic glucocorticoid dexamethasone, which readily crosses the placenta, recapitulates the major phenotypic outcomes associated with low birth weight. Thus, prenatal exposure to a dose of dexamethasone that only modestly reduces birth weight and does not affect litter size or gestational length causes permanent hypertension, hyperglycemia, hyperinsulinemia, increased hypothalamic-pituitaryadrenal (HPA) axis activity, and anxiety-like behavior in adult offspring (7-14). These effects are most marked with corticoste-

Nonstandard abbreviations used: $\operatorname{dex} 50,50 \mu \mathrm{g} / \mathrm{kg} / \mathrm{d}$ dexamethasone; $\operatorname{dex} 120$, $120 \mu \mathrm{g} / \mathrm{kg} / \mathrm{d}$ dexamethasone; dex $200,200 \mu \mathrm{g} / \mathrm{kg} / \mathrm{d}$ dexamethasone; GR, glucocorticoid receptor; HPA, hypothalamic-pituitary-adrenal; $11 \beta$-HSD2, $11 \beta$-hydroxysteroid dehydrogenase type 2; PEPCK, phosphoenolpyruvate carboxykinase; THE, tetrahydrocortisone; THF, tetrahydrocortisol.

Conflict of interest: The authors have declared that no conflict of interest exists. Citation for this article: J. Clin. Invest. 117:1058-1067 (2007). doi:10.1172/JCI30982. roid treatment in the last third of gestation (15). Similar effects of dexamethasone and related glucocorticoids have been reported in sheep, pigs, and guinea pigs (16-20). Although the precise timing of the developmental period of glucocorticoid sensitivity varies among species, the outcomes are broadly similar.

These effects of dexamethasone appear to be physiologically relevant. Treatment of pregnant rats with carbenoxolone, an inhibitor of fetoplacental $11 \beta$-hydroxysteroid dehydrogenase type 2 (11ß-HSD2), which protects the fetus from exposure to maternal physiological glucocorticoids, recapitulates the effects of dexamethasone (11). 11ß-HSD2-/- mice show reduced birth weight and programmed anxiety $(21,22)$. Similarly, chronic stress produces elevations of glucocorticoids in the mother and fetus, with a coincidental reduction in placental 11ß-HSD2 (23). Predictably, maternal stress programs cardiometabolic and neuroendocrine outcomes in the offspring $(24,25)$. It should be noted that reduced birth weight is not an obligatory consequence of prenatal glucocorticoid administration in rodents but appears mainly with lategestational exposure when fetal growth is maximal (26).

Further human studies extended the low-birth-weight baby phenotype to include altered HPA axis function later in life as an outcome (27). Interestingly, high levels of cortisol in mothers are correlated with low birth weight of their offspring (28). Further, severe stress in pregnancy (exposure to the World Trade Center attacks of September 11,2001) doubled the incidence of intrauterine growth retardation and affected salivary cortisol levels in the offspring of mothers who developed posttraumatic stress disorder; these effects were largely confined to individuals exposed in 


\section{Table 1}

Maternal plasma cortisol levels just before (12 wk gestation) and during (22 wk gestation) oral dexamethasone or vehicle (control) administration

\begin{tabular}{|c|c|c|c|c|}
\hline & $\begin{array}{c}\text { Control } \\
n=16\end{array}$ & $\begin{array}{c}\operatorname{Dex} 50 \\
n=8\end{array}$ & $\begin{array}{c}\text { Dex120 } \\
n=12\end{array}$ & $\begin{array}{c}\text { Dex200 } \\
n=16\end{array}$ \\
\hline $\begin{array}{l}\text { Cortisol levels before treatment, } \\
12 \text { wk gestation }\end{array}$ & $955 \pm 67$ & $1,102 \pm 98$ & $1,073 \pm 73$ & $1,011 \pm 88$ \\
\hline $\begin{array}{l}\text { On treatment, } 22 \text { wk gestation } \\
\text { Bonferroni versus control }\end{array}$ & $1,374 \pm 57$ & $\begin{array}{l}881 \pm 129 \\
P<0.01\end{array}$ & $\begin{array}{l}785 \pm 64 \\
P<0.001\end{array}$ & $\begin{array}{l}524 \pm 95 \\
P<0.001\end{array}$ \\
\hline
\end{tabular}

Two-way ANOVA: interaction between time and dexamethasone, $\mathrm{F}_{(3.96)}=13.48, P<0.0001$; dexamethasone, $F_{(3,96)}=9.55, P<0.0001$. There were no differences between groups before treatment (12 weeks), whereas all dexamethasone groups were significantly less than control at 22 weeks. $P<0.01$ for all dexamethasone groups (Bonferroni's multiple-comparison test).

effect on fetal heart rate, biparietal diameter, or abdominal diameter at 12 and 22 weeks of pregnancy (Table 3). Gestation length was unaffected by dexamethasone (controls, $204 \pm 5$ days from start of last menstruation; $50 \mu \mathrm{g} / \mathrm{kg} / \mathrm{d}$ dexamethasone [dex50], $204 \pm 6$ days; $120 \mu \mathrm{g} / \mathrm{kg} / \mathrm{d}$ dexamethasone [dex120], $205 \pm 4$ days; $200 \mu \mathrm{g} / \mathrm{kg} / \mathrm{d}$ dexamethasone [dex200], $200 \pm 4$ days). Fetal survival was affected by dexamethasone, but only at the highest dose; dex200 was associated with increased stillbirths (dex200, 31.3\%; control, $0 \% ; P<0.05, \chi^{2}$ test; Table 3 ).

Morphometry at birth and up to 12 months. Antenatal dexamethasone did not affect

the third trimester (29). Clinical trials involving antenatal glucocorticoid therapy have also been scrutinized retrospectively and indicate that exposure to prenatal glucocorticoids increases blood pressure in adolescence and insulin levels in adulthood $(30,31)$. However, most such studies are hampered by their retrospective nature, a lack of contemporary randomized design, and inadequate controls. Exposure to dexamethasone in the context of antenatal therapy for risk of congenital adrenal hyperplasia altered offspring behavior (in children who did not develop the disease), though birth weight was mainly unaltered $(32,33)$. However, given the efficacy of antenatal glucocorticoid therapy in such settings, prospective randomized clinical trials of any possible long-term sequelae are unlikely.

To take glucocorticoid programming closer to the human setting from the arguably removed contexts of multifetus rodent pregnancies and the dissimilar placental structure of ruminants, we have now studied the effects of antenatal glucocorticoids on the offspring in a nonhuman primate model. The vervet monkey, Chlorocebus aethiops, was used, since this Old World species produces singleton offspring and has steroid hormone biology similar to that of humans, unlike New World primates, which have a generalized steroid-resistant state.

\section{Results}

Pregnancy. Dexamethasone dose-dependently reduced the circulating levels of cortisol in maternal blood at 22 weeks of pregnancy $(P<0.0001)$, suggesting that it was absorbed and affected at least the maternal HPA axis (Table 1). However, other than suppressing maternal cortisol levels, dexamethasone had no obvious effects on the pregnant females, whose apparent well-being, as assessed by weight gain in pregnancy, maternal blood pressure, plasma electrolytes, and glucose levels, was unaffected by dexamethasone treatment (Table 2).

Dexamethasone significantly reduced fetal femoral length and, less clearly, altered placental thickness (Table 3). No dose of dexamethasone had any
Table 2

Effect of prenatal dexamethasone on maternal parameters

\begin{tabular}{|c|c|c|c|c|}
\hline & $\begin{array}{c}\text { Control } \\
n=13\end{array}$ & $\begin{array}{c}\text { Dex50 } \\
n=6\end{array}$ & $\begin{array}{c}\text { Dex120 } \\
n=13\end{array}$ & $\begin{array}{c}\text { Dex200 } \\
n=13\end{array}$ \\
\hline \multicolumn{5}{|l|}{ Maternal weight $(\mathrm{kg})$} \\
\hline Preconception & $3.60 \pm 0.18$ & $3.33 \pm 0.12$ & $3.50 \pm 0.11$ & $3.48 \pm 0.10$ \\
\hline Before treatment, 12 wk & $4.07 \pm 0.11$ & $3.90 \pm 0.16$ & $4.05 \pm 0.10$ & $4.24 \pm 0.11$ \\
\hline On treatment, 22 wk & $4.51 \pm 0.12$ & $4.48 \pm 0.25$ & $4.54 \pm 0.09$ & $4.66 \pm 0.11$ \\
\hline \multicolumn{5}{|l|}{ Maternal 24-hour urine volume (ml) } \\
\hline Before treatment, 12 wk & $71.5 \pm 9.3$ & $64.7 \pm 15.0$ & $67.3 \pm 10.7$ & $62.4 \pm 5.3$ \\
\hline On treatment, 22 wk & $71.6 \pm 10.8$ & $74.2 \pm 13.0$ & $56.4 \pm 6.1$ & $62.4 \pm 11.2$ \\
\hline \multicolumn{5}{|l|}{ Maternal blood pressure $(\mathrm{mmHg})$} \\
\hline Systolic before treatment, $12 \mathrm{wk}$ & $90.2 \pm 2.8$ & $90.3 \pm 2.5$ & $96.5 \pm 3.4$ & $98.2 \pm 5.7$ \\
\hline Systolic on treatment, 22 wk & $92.5 \pm 3.2$ & $92.1 \pm 2.3$ & $92.6 \pm 7.9$ & $97.3 \pm 3.3$ \\
\hline Diastolic before treatment, $12 \mathrm{wk}$ & $48.5 \pm 3.4$ & $46.1 \pm 4.0$ & $52.6 \pm 2.1$ & $52.7 \pm 4.3$ \\
\hline Diastolic on treatment, 22 wk & $49.6 \pm 2.4$ & $47.0 \pm 1.8$ & $54.5 \pm 2.7$ & $54.1 \pm 2.7$ \\
\hline \multicolumn{5}{|l|}{ Maternal plasma electrolytes (mM) } \\
\hline Sodium before treatment, $12 \mathrm{wk}$ & $146.0 \pm 0.7$ & $145.5 \pm 1.1$ & $143.3 \pm 0.7$ & $144.3 \pm 0.5$ \\
\hline Sodium on treatment, 22 wk & $142.2 \pm 0.7$ & $143.3 \pm 1.1$ & $142.1 \pm 0.7$ & $143.5 \pm 0.6$ \\
\hline Potassium before treatment, $12 \mathrm{wk}$ & $3.4 \pm 0.1$ & $3.7 \pm 0.3$ & $3.3 \pm 0.1$ & $3.7 \pm 0.1$ \\
\hline Potassium on treatment, 22 wk & $3.2 \pm 0.2$ & $3.0 \pm 0.1$ & $3.3 \pm 0.2$ & $3.6 \pm 0.2$ \\
\hline Chloride before treatment, $12 \mathrm{wk}$ & $108.9 \pm 0.9$ & $106.8 \pm 3.3$ & $108.2 \pm 0.6$ & $109.4 \pm 0.6$ \\
\hline Chloride on treatment, 22 wk & $109.1 \pm 1.0$ & $106.8 \pm 1.4$ & $107.2 \pm 0.7$ & $108.9 \pm 0.7$ \\
\hline
\end{tabular}

No statistically significant differences. birth weight or the ponderal index (Table 4). Similarly, at birth, crown-heel length, crown-rump length, abdominal circumference, head circumference, biparietal diameter, head length, forearm length, femur and tibia length, hip width, and subcutaneous fat thickness were all unaltered by antenatal dexamethasone (Table 4). In contrast to the lack of effects on morphometric parameters at birth, antenatal dexamethasone exposure did alter postnatal growth in the majority of the estimated parameters (Table 4). In general, antenatal dexamethasone attenuated growth (length, weight) and increased subcutaneous fat thickness.

Neonatal behavior. The Brazelton score is an index for newborns and very young children that estimates the baby's strength, adaptive responses, and possible vulnerabilities. Here we used an adapted Brazelton score that gives a measure for palmar and plantar grasp response, body righting, resistance to passive movement, active power, tactile response, spontaneous locomotion, and motor activity (adapted from ref. 34). The adapted Brazelton scores were similar in all offspring and were unaffected by ante- 
natal glucocorticoids (Table 5). Kruskal-Wallis testing supported a diminished motor activity at a higher dose of prenatal dexamethasone $(P=0.007)$.

Gross measurements at 12-14 months. A subset of animals was sacrificed at 12-14 months of age. Organ weights (gross as well as relative to total body weight) of the left and right adrenals, thymus, left and right kidneys, heart, and brain were not affected by antenatal glucocorticoid exposure. Pituitary weight was increased in dex200 offspring ( $P<0.05$ versus control; data not shown).

Insulin and glucose. Oral glucose tolerance tests were performed in 8-month-old offspring after an overnight fast. Offspring of dex120 pregnancies had higher fasting plasma insulin levels (Figure 1). Insulin increased to greater extent after glucose tolerance tests in dex120 and dex200 offspring as compared with controls (Figure 1). Fasting and peak glucose levels were similar in all groups, but subsequent glucose clearance was significantly slower in the dex120 and dex200 offspring (Figure 1), and there was a similar trend in the dex50 offspring $(P=0.051)$.

Pancreas. There was a pronounced reduction in the number of $\beta$ cells compared with total cell number ( $\alpha$ and $\beta$ cell number combined) in the pancreatic islets of 12- to 14-month-old offspring of dex120 and dex200 pregnancies compared with controls (Figure 2). Moreover, dex 120 and dex 200 offspring showed smaller insulin-producing $\beta$ cells than controls (controls, $116 \pm 11 \mu \mathrm{m}^{2}$; dex50, $90 \pm 22$ $\mu \mathrm{m}^{2}$; dex120, $52 \pm 3 \mu \mathrm{m}^{2}$; $\operatorname{dex} 200,48 \pm 2 \mu \mathrm{m}^{2} ; P<0.002$ ), while $\alpha$ cell size was unaltered (controls, $146 \pm 26 \mu \mathrm{m}^{2}$; dex50, $106 \pm 23 \mu \mathrm{m}^{2}$; $\operatorname{dex} 120,141 \pm 5 \mu \mathrm{m}^{2}$; $\operatorname{dex} 200,142 \pm 3 \mu \mathrm{m}^{2}$ ), resulting in a smaller $\beta$ cell $/ \alpha$ cell area ratio. Dex 50 had no effect on $\beta$ cell parameters.

Liver. The key hepatic gluconeogenic enzyme phosphoenolpyruvate carboxykinase (PEPCK) and one of its major regulating transcription factors, the glucocorticoid receptor (GR), were estimated on the basis of previous rodent findings with antenatal dexamethasone (26). PEPCK activity as well as mRNA was reduced in dex120 and dex200 offspring livers (Figure 3), and, as expected, PEPCK mRNA and activity were correlated $(r=0.63, P=0.01)$. PEPCK mRNA and GR mRNA contents in the liver were also interrelated and affected by the different prenatal dexamethasone treatments (Figure 3; 2-way ANOVA for PEPCK and GR mRNA, $\mathrm{F}_{(3,12)}=3.96$; interaction between offspring groups and PEPCK and GR mRNA, $P=0.036$ ).

Blood pressure. Blood pressure was measured in the offspring of control and dexamethasone-treated mothers at 8 and again at 12-14 months of age. Whereas systolic and diastolic pressures were similar in all groups at 8 months (Figure 4), by $12-14$ months dex 120 and dex 200 offspring showed a significant increase in systolic (+12 and $+17 \mathrm{mmHg}$, respectively) and diastolic (+18 and $+17 \mathrm{mmHg}$, respectively) pressure as compared with controls (Figure 4). Dex50 offspring showed a small but significant decrease in systolic pressure (by $9 \mathrm{mmHg}$ ) at $12-14$ months, but diastolic pressure was unaffected.

Cortisol and its metabolites. Mild stress, induced by blood sampling, produced an elevation of the plasma cortisol levels to a significantly greater extent in dex200 offspring than in controls (Figure 5). To test negative feedback, infants at 12-14 months were given a dexamethasone suppression test, but this showed no difference in morning cortisol levels between controls and different prenatal dexamethasone treatments (plasma cortisol levels 9 hours after $0.026 \mathrm{mg} / \mathrm{kg}$ dexamethasone given at 7 am were: controls, $1,887 \pm 168 \mathrm{nmol} / 1$; dex $50,1,501 \pm 258 \mathrm{nmol} / \mathrm{l}$; dex120, $1,387 \pm 146 \mathrm{nmol} / \mathrm{l}$; and dex200, 1,736 $\pm 227 \mathrm{nmol} / \mathrm{l}$; NS).

Tissue metabolism of cortisol may alter plasma levels. However, 24-hour urinary collections and gas chromatographic/mass spectrometric analysis revealed no group differences in the levels of cortisol, cortisone, tetrahydrocortisols, tetrahydrocortisone,

\section{Table 3}

Fetal parameters just before (12 wk gestation) and during (22 wk gestation) oral dexamethasone or vehicle (control) administration

\begin{tabular}{|c|c|c|c|c|c|c|}
\hline & $\begin{array}{c}\text { Control } \\
n=13\end{array}$ & $\begin{array}{c}\operatorname{Dex} 50 \\
n=6\end{array}$ & $\begin{array}{c}\text { Dex120 } \\
n=13\end{array}$ & $\begin{array}{c}\text { Dex200 } \\
n=13\end{array}$ & $\begin{array}{l}\text { Interaction time/dex } \\
\qquad \mathrm{F}_{(3,41)}\end{array}$ & $\begin{array}{l}\text { Dex } \\
\mathrm{F}_{(3,41)}\end{array}$ \\
\hline Placental thickness (mm) & & & & & $3.52^{\mathrm{A}}$ & NS \\
\hline No dex, 12 wk & $9.8 \pm 0.4$ & $10.7 \pm 0.6$ & $10.2 \pm 0.4$ & $9.7 \pm 0.5$ & & \\
\hline On dex, 22 wk & $14.0 \pm 0.8$ & $13.3 \pm 0.6$ & $13.2 \pm 0.7$ & $15.3 \pm 0.5$ & & \\
\hline Fetal heart rate (bpm) & & & & & NS & NS \\
\hline No dex, 12 wk & $154 \pm 3$ & $161 \pm 2$ & $171 \pm 7$ & $173 \pm 5$ & & \\
\hline On dex, 22 wk & $159 \pm 2$ & $155 \pm 4$ & $164 \pm 8$ & $169 \pm 6$ & & \\
\hline Biparietal diameter (mm) & & & & & NS & NS \\
\hline No dex, 12 wk & $26.1 \pm 0.9$ & $25.7 \pm 0.7$ & $26.5 \pm 1.2$ & $28.4 \pm 1.0$ & & \\
\hline On dex, 22 wk & $41.0 \pm 0.7$ & $43.5 \pm 0.3$ & $43.4 \pm 0.6$ & $43.1 \pm 0.5$ & & \\
\hline Abdominal diameter (mm) & & & & & NS & NS \\
\hline No dex, 12 wk & $19.1 \pm 0.9$ & $20.5 \pm 0.8$ & $17.9 \pm 0.7$ & $18.4 \pm 0.9$ & & \\
\hline On dex, 22 wk & $31.7 \pm 0.9$ & $29.5 \pm 1.7$ & $32.8 \pm 0.9$ & $29.7 \pm 1.1$ & & \\
\hline Femur length (mm) & & & & & $4.26^{A}$ & $3.16^{A}$ \\
\hline No dex, 12 wk & $14.1 \pm 0.6$ & $14.8 \pm 0.8$ & $14.2 \pm 0.7$ & $14.5 \pm 0.7$ & & \\
\hline On dex, 22 wk & $41.1 \pm 2.1$ & $39.8 \pm 3.3$ & $31.2 \pm 2.9 \mathrm{~A}$ & $31.7 \pm 2.5^{\mathrm{A}}$ & & \\
\hline Fetal survival & & & & & & \\
\hline Abortion no. ( $\%$ of total live births) & $1(6.7 \%)$ & $0(0 \%)$ & $0(0 \%)$ & $2(12.5 \%)$ & & \\
\hline Stillbirth no. ( $\%$ of total live births) & $0(0 \%)$ & $1(16.7 \%)$ & $1(7.7 \%)$ & $5(31.3 \%)^{A}$ & & \\
\hline Live birth no. (\% of total pregnancies) & $16(93.8 \%)$ & $6(85.7 \%)$ & $13(92.9 \%)$ & $16(69.6 \%)$ & & \\
\hline No. alive at $12 \mathrm{mo}$ (\% of live births) & $13(87.7 \%)$ & $6(100 \%)$ & $13(100 \%)$ & $13(81.3 \%)$ & & \\
\hline
\end{tabular}


Table 4

Effects of prenatal maternal exposure to oral dexamethasone or vehicle (control) on anthropometric parameters in newborns and infants

\begin{tabular}{|c|c|c|c|c|c|c|}
\hline & $\begin{array}{c}\text { Control } \\
n=13\end{array}$ & $\begin{array}{c}\text { Dex50 } \\
n=6\end{array}$ & $\begin{array}{c}\text { Dex120 } \\
n=13\end{array}$ & $\begin{array}{c}\text { Dex200 } \\
n=13\end{array}$ & $\begin{array}{l}\text { Interaction time/dex } \\
\mathbf{F}_{(15,205)}\end{array}$ & $\begin{array}{c}\text { Dex } \\
F_{(3,205)}\end{array}$ \\
\hline Weight (g) & & & & & $2.87^{\mathrm{A}}$ & NS \\
\hline $0 \mathrm{mo}$ & $323 \pm 9$ & $350 \pm 18$ & $320 \pm 9$ & $308 \pm 12$ & & \\
\hline $2 \mathrm{mo}$ & $568 \pm 21$ & $605 \pm 30$ & $571 \pm 28$ & $551 \pm 37$ & & \\
\hline $4 \mathrm{mo}$ & $692 \pm 28$ & $727 \pm 26$ & $760 \pm 24$ & $699 \pm 32$ & & \\
\hline $6 \mathrm{mo}$ & $852 \pm 35$ & $823 \pm 16$ & $875 \pm 31$ & $862 \pm 25$ & & \\
\hline $8 \mathrm{mo}$ & $1,066 \pm 33$ & $1,033 \pm 17$ & $1,001 \pm 33^{B}$ & $1,002 \pm 31^{B}$ & & \\
\hline $12 \mathrm{mo}$ & $1,395 \pm 50$ & $1,347 \pm 64$ & $1,308 \pm 37^{B}$ & $1,215 \pm 44^{B}$ & & \\
\hline Head circumference (mm) & & & & & NS & NS \\
\hline $0 \mathrm{mo}$ & $174 \pm 2$ & $177 \pm 3$ & $173 \pm 3$ & $172 \pm 2$ & & \\
\hline $2 \mathrm{mo}$ & $196 \pm 2$ & $200 \pm 3$ & $192 \pm 3$ & $194 \pm 2$ & & \\
\hline $4 \mathrm{mo}$ & $199 \pm 6$ & $201 \pm 4$ & $199 \pm 2$ & $199 \pm 2$ & & \\
\hline $6 \mathrm{mo}$ & $207 \pm 5$ & $205 \pm 3$ & $203 \pm 2$ & $204 \pm 2$ & & \\
\hline $8 \mathrm{mo}$ & $207 \pm 2$ & $207 \pm 2$ & $208 \pm 2$ & $206 \pm 3$ & & \\
\hline $12 \mathrm{mo}$ & $212 \pm 2$ & $212 \pm 3$ & $209 \pm 2$ & $209 \pm 3$ & & \\
\hline Head length (mm) & & & & & NS & NS \\
\hline $0 \mathrm{mo}$ & $59.1 \pm 0.4$ & $59.0 \pm 1.3$ & $58.6 \pm 1.0$ & $57.2 \pm 0.5$ & & \\
\hline $2 \mathrm{mo}$ & $65.3 \pm 0.6$ & $66.8 \pm 0.8$ & $64.2 \pm 0.9$ & $63.5 \pm 0.8$ & & \\
\hline $4 \mathrm{mo}$ & $66.8 \pm 0.7$ & $68.8 \pm 1.2$ & $66.3 \pm 0.8$ & $65.3 \pm 0.8$ & & \\
\hline $6 \mathrm{mo}$ & $68.2 \pm 0.5$ & $68.1 \pm 0.6$ & $67.6 \pm 0.8$ & $67.0 \pm 0.7$ & & \\
\hline $8 \mathrm{mo}$ & $70.0 \pm 0.9$ & $69.5 \pm 0.4$ & $68.5 \pm 0.7$ & $67.8 \pm 0.8$ & & \\
\hline $12 \mathrm{mo}$ & $70.0 \pm 0.8$ & $70.2 \pm 0.6$ & $70.0 \pm 0.8$ & $69.5 \pm 0.8$ & & \\
\hline Biparietal diameter (mm) & & & & & NS & NS \\
\hline $0 \mathrm{mo}$ & $45.7 \pm 0.8$ & $46.9 \pm 0.4$ & $46.7 \pm 0.5$ & $46.1 \pm 0.5$ & & \\
\hline $2 \mathrm{mo}$ & $53.8 \pm 0.3$ & $55.1 \pm 0.6$ & $53.5 \pm 0.8$ & $53.5 \pm 0.7$ & & \\
\hline $4 \mathrm{mo}$ & $55.2 \pm 0.5$ & $55.6 \pm 0.8$ & $56.0 \pm 0.6$ & $55.4 \pm 0.7$ & & \\
\hline $6 \mathrm{mo}$ & $56.7 \pm 0.5$ & $57.5 \pm 0.7$ & $57.5 \pm 0.5$ & $57.2 \pm 0.6$ & & \\
\hline $8 \mathrm{mo}$ & $56.5 \pm 0.9$ & $56.9 \pm 0.6$ & $57.6 \pm 0.5$ & $57.8 \pm 0.7$ & & \\
\hline $12 \mathrm{mo}$ & $57.4 \pm 0.5$ & $57.7 \pm 0.6$ & $58.9 \pm 0.5$ & $58.7 \pm 0.6$ & & \\
\hline Crown-heel length (mm) & $01.4 \pm 0.0$ & & 00.010 .0 & $00.1 \pm 0.0$ & $1.76^{\mathrm{B}}$ & $4.19^{\mathrm{B}}$ \\
\hline $0 \mathrm{mo}$ & $272 \pm 7$ & $290 \pm 6$ & $276 \pm 4$ & $270 \pm 4$ & & \\
\hline $2 \mathrm{mo}$ & $343 \pm 8$ & $366 \pm 6$ & $346 \pm 7$ & $346 \pm 8$ & & \\
\hline $4 \mathrm{mo}$ & $393 \pm 7$ & $415 \pm 10$ & $384 \pm 7$ & $380 \pm 8$ & & \\
\hline $6 \mathrm{mo}$ & $430 \pm 8$ & $445 \pm 8$ & $412 \pm 8$ & $404 \pm 7^{8}$ & & \\
\hline $8 \mathrm{mo}$ & $462 \pm 7$ & $463 \pm 6$ & $432 \pm 8^{B}$ & $436 \pm 8^{B}$ & & \\
\hline $12 \mathrm{mo}$ & $498 \pm 9$ & $513 \pm 8$ & $483 \pm 5$ & $475 \pm 7^{B}$ & & \\
\hline Crown-rump length (mm) & & & & & NS & NS \\
\hline $0 \mathrm{mo}$ & $190 \pm 3$ & $194 \pm 4$ & $183 \pm 3$ & $178 \pm 3$ & & \\
\hline $2 \mathrm{mo}$ & $236 \pm 4$ & $252 \pm 10$ & $230 \pm 5$ & $229 \pm 5$ & & \\
\hline $4 \mathrm{mo}$ & $265 \pm 5$ & $270 \pm 5$ & $261 \pm 4$ & $261 \pm 5$ & & \\
\hline $6 \mathrm{mo}$ & $285 \pm 7$ & $296 \pm 2$ & $279 \pm 3$ & $286 \pm 3$ & & \\
\hline $8 \mathrm{mo}$ & $305 \pm 6$ & $307 \pm 1$ & $296 \pm 3$ & $306 \pm 4$ & & \\
\hline $12 \mathrm{mo}$ & $330 \pm 5$ & $339 \pm 6$ & $330 \pm 4$ & $322 \pm 4$ & & \\
\hline Abdominal circumference (mm) & & & & & NS & NS \\
\hline 0 mo & $123 \pm 3$ & $122 \pm 3$ & $119 \pm 2$ & $117 \pm 3$ & & \\
\hline $2 \mathrm{mo}$ & $141 \pm 5$ & $142 \pm 3$ & $138 \pm 2$ & $140 \pm 3$ & & \\
\hline $4 \mathrm{mo}$ & $153 \pm 4$ & $156 \pm 6$ & $155 \pm 4$ & $154 \pm 3$ & & \\
\hline $6 \mathrm{mo}$ & $162 \pm 5$ & $172 \pm 9$ & $167 \pm 4$ & $169 \pm 3$ & & \\
\hline $8 \mathrm{mo}$ & $179 \pm 4$ & $181 \pm 3$ & $180 \pm 4$ & $182 \pm 3$ & & \\
\hline $12 \mathrm{mo}$ & $212 \pm 5$ & $206 \pm 4$ & $202 \pm 4$ & $198 \pm 3$ & & \\
\hline Subcutaneous fat (mm) & & & & & $2.62^{\mathrm{C}}$ & $4.04^{B}$ \\
\hline $0 \mathrm{mo}$ & $0.83 \pm 0.09$ & $0.77 \pm 0.13$ & $0.80 \pm 0.10$ & $0.71 \pm 0.05$ & & \\
\hline $2 \mathrm{mo}$ & $1.07 \pm 0.12$ & $1.11 \pm 0.09$ & $0.99 \pm 0.07$ & $0.87 \pm 0.07$ & & \\
\hline $4 \mathrm{mo}$ & $1.08 \pm 0.12$ & $0.90 \pm 0.17$ & $1.00 \pm 0.05$ & $0.87 \pm 0.03$ & & \\
\hline $6 \mathrm{mo}$ & $0.92 \pm 0.08$ & $1.00 \pm 0.10$ & $1.15 \pm 0.07^{B}$ & $1.15 \pm 0.10^{\mathrm{B}}$ & & \\
\hline $8 \mathrm{mo}$ & $0.82 \pm 0.07$ & $0.97 \pm 0.09$ & $1.26 \pm 0.09^{B}$ & $1.15 \pm 0.04^{B}$ & & \\
\hline $12 \mathrm{mo}$ & $0.97 \pm 0.13$ & $0.67 \pm 0.04^{B}$ & $1.25 \pm 0.07^{B}$ & $1.19 \pm 0.06$ & & \\
\hline Femur length (mm) & & & & & $1.88^{\mathrm{B}}$ & $7.83^{\mathrm{A}}$ \\
\hline $0 \mathrm{mo}$ & $59.4 \pm 1.1$ & $60.7 \pm 1.6$ & $59.6 \pm 3.2$ & $55.7 \pm 1.0$ & & \\
\hline $2 \mathrm{mo}$ & $80.1 \pm 2.1$ & $83.3 \pm 3.5$ & $71.3 \pm 1.4^{\mathrm{B}}$ & $74.9 \pm 1.8$ & & \\
\hline $4 \mathrm{mo}$ & $84.6 \pm 2.0$ & $89.1 \pm 1.8$ & $78.9 \pm 1.5^{B}$ & $80.4 \pm 1.7$ & & \\
\hline $6 \mathrm{mo}$ & $95.9 \pm 2.5$ & $95.4 \pm 2.2$ & $86.1 \pm 1.9 \mathrm{~B}$ & $85.0 \pm 2.2^{B}$ & & \\
\hline $8 \mathrm{mo}$ & $102.2 \pm 2.6$ & $100.5 \pm 2.2$ & $91.1 \pm 2.1^{\mathrm{B}}$ & $91.5 \pm 2.2^{B}$ & & \\
\hline $12 \mathrm{mo}$ & $107.0 \pm 1.9$ & $110.1 \pm 2.1$ & $103.0 \pm 1.7$ & $100.2 \pm 2.3^{\mathrm{B}}$ & & \\
\hline Tibia length (mm) & & & & & $2.19 \mathrm{c}$ & NS \\
\hline 0 mo & $67.0 \pm 1.0$ & $70.4 \pm 1.5$ & $69.1 \pm 1.1$ & $67.5 \pm 0.8$ & & \\
\hline $2 \mathrm{mo}$ & $88.6 \pm 1.2$ & $91.1 \pm 2.3$ & $85.9 \pm 2.1$ & $87.2 \pm 1.9$ & & \\
\hline $4 \mathrm{mo}$ & $97.3 \pm 2.0$ & $97.4 \pm 0.8$ & $94.5 \pm 1.7$ & $95.0 \pm 1.5$ & & \\
\hline $6 \mathrm{mo}$ & $106.2 \pm 2.2$ & $108.6 \pm 0.9$ & $101.7 \pm 2.1$ & $103.8 \pm 1.8$ & & \\
\hline $8 \mathrm{mo}$ & $114.5 \pm 2.4$ & $114.2 \pm 1.4$ & $107.5 \pm 2.4^{B}$ & $110.3 \pm 1.8$ & & \\
\hline $12 \mathrm{mo}$ & $125.3 \pm 2.1$ & $127.0 \pm 2.3$ & $121.5 \pm 2.3$ & $117.1 \pm 2.2^{\mathrm{B}}$ & & \\
\hline Forearm length $(\mathrm{mm})$ & & & & & $3.21^{\mathrm{A}}$ & NS \\
\hline $0 \mathrm{mo}$ & $57.7 \pm 0.8$ & $61.3 \pm 1.3$ & $62.1 \pm 2.4^{B}$ & $58.6 \pm 0.8$ & & \\
\hline $2 \mathrm{mo}$ & $73.0 \pm 1.0$ & $75.2 \pm 1.4$ & $72.9 \pm 1.4$ & $74.1 \pm 1.0$ & & \\
\hline $4 \mathrm{mo}$ & $80.6 \pm 2.1$ & $80.4 \pm 1.2$ & $80.3 \pm 1.6$ & $78.9 \pm 0.8$ & & \\
\hline $6 \mathrm{mo}$ & $86.9 \pm 1.8$ & $88.7 \pm 1.1$ & $84.6 \pm 1.7$ & $83.5 \pm 1.3$ & & \\
\hline $8 \mathrm{mo}$ & $92.9 \pm 1.9$ & $94.5 \pm 1.3$ & $89.1 \pm 1.9$ & $88.2 \pm 1.7^{B}$ & & \\
\hline $12 \mathrm{mo}$ & $104.4 \pm 1.7$ & $106.3 \pm 2.7$ & $99.5 \pm 1.5^{\mathrm{B}}$ & $95.1 \pm 2.1^{\mathrm{B}}$ & & \\
\hline Hip width (mm) & & & & & $1.94^{\mathrm{B}}$ & NS \\
\hline $0 \mathrm{mo}$ & $27.9 \pm 0.8$ & $29.6 \pm 1.5$ & $28.2 \pm 0.6$ & $26.5 \pm 0.6$ & & \\
\hline $2 \mathrm{mo}$ & $34.3 \pm 0.7$ & $34.3 \pm 1.3$ & $34.2 \pm 0.7$ & $33.8 \pm 0.8$ & & \\
\hline $4 \mathrm{mo}$ & $37.2 \pm 0.6$ & $36.2 \pm 0.8$ & $37.0 \pm 0.7$ & $36.2 \pm 0.6$ & & \\
\hline $6 \mathrm{mo}$ & $40.7 \pm 0.6$ & $38.7 \pm 0.7$ & $40.0 \pm 0.7$ & $39.0 \pm 0.6$ & & \\
\hline $8 \mathrm{mo}$ & $42.0 \pm 0.9$ & $40.7 \pm 0.8$ & $41.4 \pm 0.7$ & $41.2 \pm 0.6$ & & \\
\hline $12 \mathrm{mo}$ & $46.6 \pm 1.0$ & $45.0 \pm 0.6$ & $44.9 \pm 0.5$ & $43.4 \pm 0.6^{B}$ & & \\
\hline Ponderal index & $1.56 \pm 0.07$ & $1.14 \pm 0.03$ & $1.52 \pm 0.04$ & $1.57 \pm 0.05$ & NS & NS \\
\hline
\end{tabular}

Significant differences from controls are shown in boldface. ${ }^{A} P<0.001, B P<0.05, C P<0.01$ versus control. Last 2 columns: 2 -way repeated-measures ANOVA. In cells, post hoc testing by Fisher's least significant difference method for factor versus control. 


\section{Table 5}

Neurodevelopmental effects of antenatal exposure to dexamethasone or vehicle as reflected in Brazelton scores in neonatal offspring

\begin{tabular}{lcccc}
\hline & $\begin{array}{c}\text { Control } \\
\boldsymbol{n}=\mathbf{1 3}\end{array}$ & $\begin{array}{c}\text { Dex50 } \\
\boldsymbol{n}=\mathbf{6}\end{array}$ & $\begin{array}{c}\text { Dex120 } \\
\boldsymbol{n}=\mathbf{1 2}\end{array}$ & $\begin{array}{c}\text { Dex200 } \\
\boldsymbol{n}=\mathbf{1 1}\end{array}$ \\
& $1.92 \pm 0.08$ & $2.00 \pm 0.00$ & $1.92 \pm 0.08$ & $1.91 \pm 0.09$ \\
Palmar grasp response & $1.92 \pm 0.08$ & $1.83 \pm 0.17$ & $1.83 \pm 0.11$ & $1.91 \pm 0.09$ \\
Plantar grasp response & $1.85 \pm 0.15$ & $1.67 \pm 0.33$ & $1.83 \pm 0.17$ & $1.64 \pm 0.20$ \\
Body righting & $1.77 \pm 0.12$ & $2.00 \pm 0.00$ & $1.67 \pm 0.19$ & $1.82 \pm 0.18$ \\
Resistance to passive movement & $1.77 \pm 0.12$ & $2.00 \pm 0.00$ & $1.33 \pm 0.23$ & $1.64 \pm 0.20$ \\
Active power & $2.00 \pm 0.00$ & $2.00 \pm 0.00$ & $1.92 \pm 0.83$ & $2.00 \pm 0.00$ \\
Tactile response & $1.31 \pm 0.21$ & $1.00 \pm 0.26$ & $1.58 \pm 0.19$ & $1.73 \pm 0.41$ \\
Spontaneous locomotion & $2.00 \pm 0.00$ & $2.00 \pm 0.00$ & $1.42 \pm 0.19 \mathrm{~A}$ & $1.73 \pm 0.41$ \\
Motor activity & & & &
\end{tabular}

Kruskal-Wallis testing shows diminished motor activity in offspring following the higher doses of prenatal dexamethasone; $P=0.007$. Mann-Whitney $U$ post hoc testing, ${ }^{A} P<0.05$.

effects of glucocorticoids on placental size have been noted previously (39) but are of uncertain significance. The highest dose of dexamethasone increased stillbirth, mirroring anecdotal human reports $(40,41)$ and findings in monkeys (42).

Intriguingly, weight and other parameters at birth were also unaltered by dexamethasone. This contrasts with several rodent programming models using dexamethasone, stress, and $11 \beta$-HSD2 inhibition, all of which reduce birth weight (15, $22,25)$. However, mid- to late-gestational stress in rhesus monkeys also fails to alter birth weight (43), and guinea pigs resist changes in birth weight following maternal food restriction, though this stimulus has programming effects on offspring's

cortolones, or cortols (data not shown). No ratios that reflect $5 \alpha$ or $5 \beta$-reduction or $11 \beta$-hydroxysteroid dehydrogenation were altered by prenatal dexamethasone in any dose.

\section{Discussion}

Here we show that maternal dexamethasone treatment causes persistent cardiometabolic and neuroendocrine effects in the offspring in nonhuman primates. The effects are broadly similar to those seen in rodents and suggest that antenatal glucocorticoid therapy may also have persistent effects in primates. The effects of dexamethasone were dose-associated. The lack of effect of dexamethasone at any dose on birth weight parallels some human data $(35,36)$ and suggests that, in primates, fetal programming by glucocorticoids may occur independently of large changes in anthropometric indices at birth.

Fetal growth and birth parameters. Prenatal dexamethasone had no discernible effects on most fetal parameters. However, femur length was reduced by dexamethasone - length has distinct associations in the human epidemiological canon, notably with lifespan and cardiovascular disorders (37) - demonstrating at least 1 fetal growth-retarding action, as previously reported in primates (38). Placental thickness was also altered by dexamethasone, though lower doses tended to thin the placenta whereas dex200 thickened it; such dose-related inconsistent

\section{Figure 1}

Intravenous glucose tolerance tests in offspring of dexamethasone- and control-treated pregnancies. (A-C) Plasma glucose levels before (time $=0$ ) and during an i.v. glucose tolerance test. Overall glucose clearance was affected by antenatal dexamethasone treatment (2-way ANOVA, $\left.\mathrm{F}_{(3,244)}=4.4, P=0.005\right)$. The glucose clearance was slower in the dex120 and dex200 offspring than in controls (post hoc testing, Fisher's least significant difference method for factor, ${ }^{*} P<0.02$ for dex120 and dex200; $P=0.051$ for dex50). (D) Insulin levels of 8-month-old offspring before and 10 minutes after i.v. glucose administration. Basal insulin levels were increased in dex120 offspring $\left({ }^{\dagger} P<0.05\right.$ versus control; 2 -tailed Student's $t$ test). At 10 minutes, plasma insulin was elevated in dex120 and dex200 offspring ( ${ }^{\dagger} P<0.05$ versus control; 2-tailed Student's $t$ test).
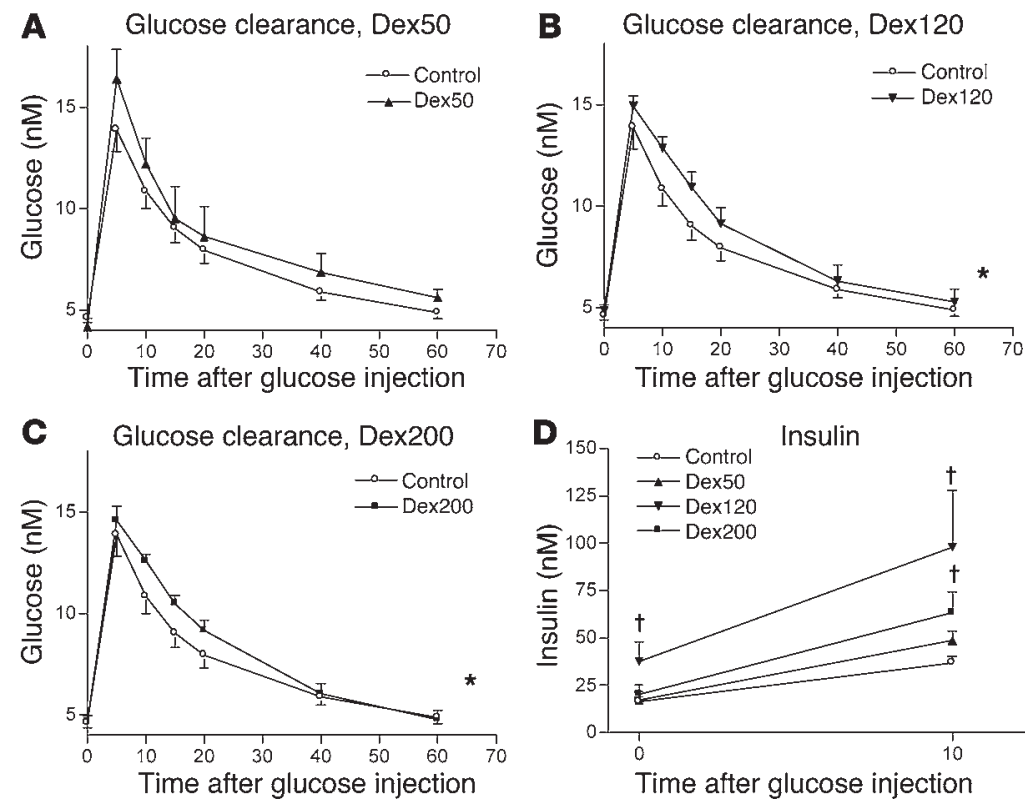


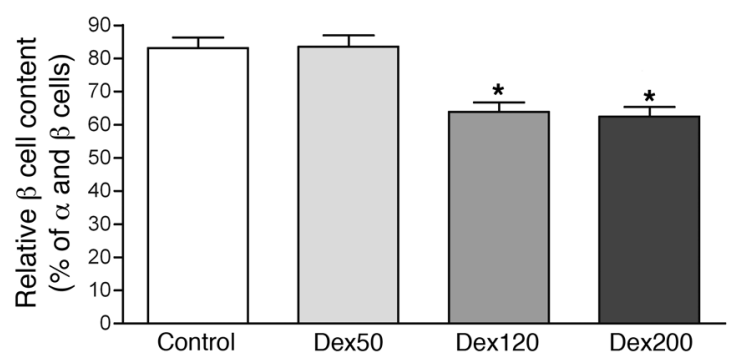

Figure 2

Pancreatic $\beta$ cell content is reduced in the offspring of dexamethasonetreated pregnancies. The $\beta$ cell content relative to the total of $\alpha$ and $\beta$ cells in the pancreatic islets is depicted. One-way ANOVA, $\mathrm{F}_{(3,23)}=18.47$, $P<0.0001$; Bonferroni's multiple-comparison test, ${ }^{\star} P<0.001$.

Previous reports document that antenatal stress or glucocorticoid exposure alters offspring behavior and CNS structure (49-51). While, at birth, behavior was largely similar in control and dexamethasone-exposed offspring, the motor subset of Brazelton scores showed significant retardation in dexamethasone-exposed offspring. Such findings parallel motor retardation seen in earlypostnatal $11 \beta-\mathrm{HSD}_{2}{ }^{--}$mice (52) but have not been reported, at least as determined by maternal questionnaire, following low-dose dexamethasone in early gestation in antenatal therapy for congenital adrenal hyperplasia risk (53). Since the Brazelton score is a clinical tool based on sensorimotor function at birth, the data here do not confirm that antenatal dexamethasone in late gestation has no effects on the developing primate CNS. However, the Brazelton score has limited predictive value, and it would be interesting to reanalyze sensorimotor function later in life.

Prenatal dexamethasone and later pathophysiology. While prenatal dexamethasone had only a few, relatively subtle actions on morphology at birth, thereafter persisting effects were clear-cut. Both dex120 and dex200 attenuated postnatal growth and increased subcutaneous fat. There were also clear effects on glucose-insulin homeostasis, pancreatic endocrine structure, blood pressure, and HPA axis activity. However subtle, these effects were detectable even in very young animals. The effects of early-life manipulations in rodents and the birth weight observations in humans suggest amplification of phenotypes with aging and with adult challenges, notably obesity (54).

Abnormal glucose tolerance was observed in dexamethasonetreated offspring, notably, delayed glucose clearance, likely mediated by reduced peripheral glucose uptake. This was not due to frank obesity; indeed, the dexamethasone-treated offspring were lighter, though increased fat mass may have contributed (juvenile monkeys are very lean). As well as delayed glucose clearance, in rats (26) and sheep (55) fasting hyperglycemia is reported after antenatal dexamethasone, stress, or $11 \beta$-hydroxysteroid dehydrogenase inhibition. This was not seen here, but the monkeys are prepubertal, and perhaps other defects in glucose tolerance will become manifest as they age, as observed in some rodent models (56). The data here suggest that delayed glucose clearance is an early abnormality in dexamethasone-programmed primates. The increased insulin release in response to glucose challenge in dex120 and dex 200 offspring supports the notion of early insulin resistance. However, the reduction of pancreatic $\beta$ cell number and size at 1 year suggests that the extent of any compensatory hyperinsulinemia will be limited, risking type 2 diabetes over and above any later loss of $\beta$ cell function (57). Interestingly, fasting insulin levels rose in response to dextrose loading in dex120 but not in dex 200 offspring (Figure 1), which may point to an earlier $\beta$ cell decompensation following higher-dose exposure. Antenatal glucocorticoids have been previously reported to reduce $\beta$ cell mass in adult mice (58) and rats (10), and in rhesus fetuses (59), in line with our finding in juvenile vervets. Intriguingly, brief late-gestational exposure to glucocorticoids in humans associates with postglucose hyperinsulinemia more than 3 decades later (31).

In previous rat studies, hepatic PEPCK was increased in the offspring of dexamethasone-exposed pregnancies $(9,26)$. In young vervets, we find hepatic PEPCK is reduced after antenatal dexamethasone. PEPCK gene regulation is complex and involves interplay among insulin, glucocorticoids, and other counterregulatory hormones (60). In rats, several transcription factors involved in PEPCK control are altered by antenatal dexamethasone (61). We suggest that the changes in PEPCK in vervet offspring reflect this interplay and that reduced PEPCK may ameliorate any tendency toward fasting hyperglycemia in these animals. GR has been proposed as a key target for antenatal glucocorticoid or nutritional

\section{Figure 3}

PEPCK activity in the liver is diminished in the offspring of pregnant animals given dexamethasone, coinciding with attenuated PEPCK mRNA and altered GR mRNA expression. Top left: PEPCK activity in the liver. One-way ANOVA, $P=0.0096$; Newman-Keuls multiple-comparison test, ${ }^{*} P<0.05$ versus control. Bottom left: Relative PEPCK mRNA content in the liver. Bottom right: Relative GR mRNA content in the liver. Two-way ANOVA for PEPCK and GR mRNA, $F_{(3,12)}=3.96$; interaction between PEPCK and GR mRNA, $P=0.036$.
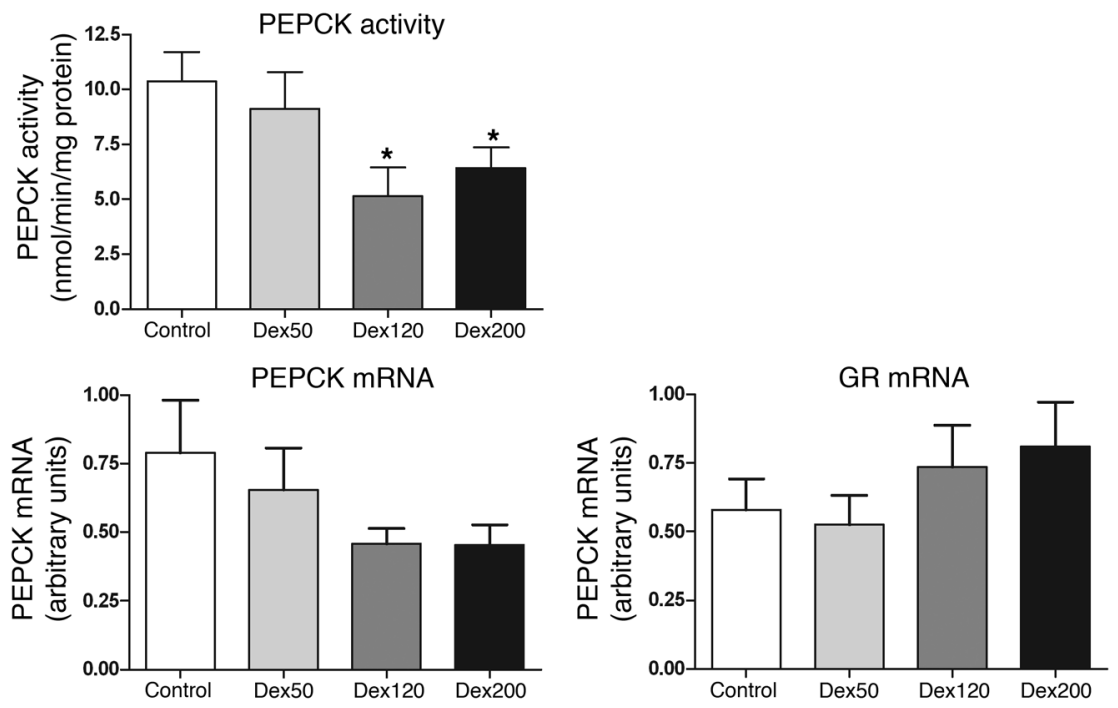

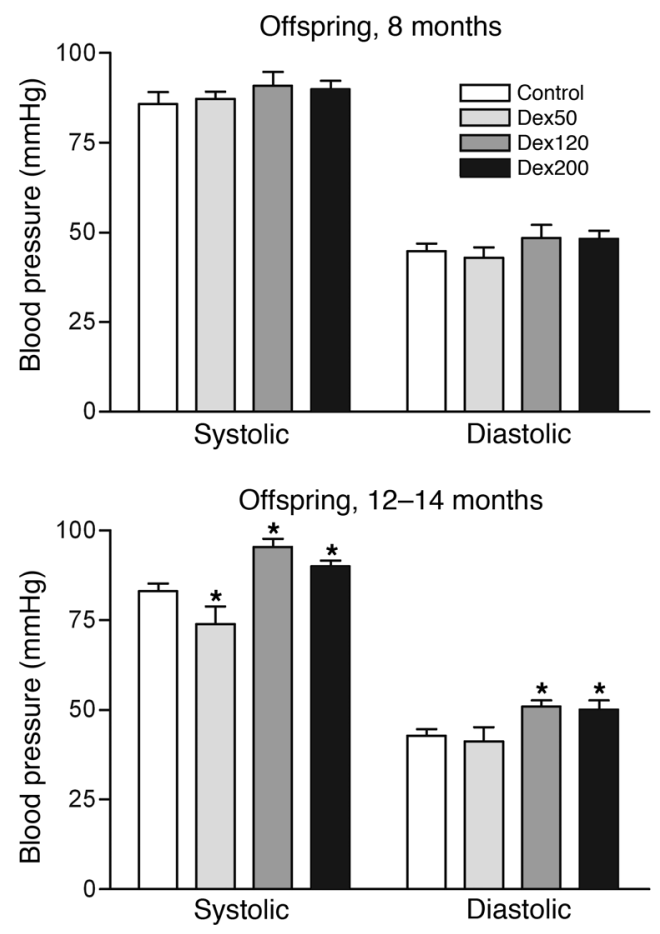

programming $(62,63)$. The trend of increased GR in liver here, paralleling similar significant findings in rats, suggests that similar broad principles operate in primates. Indeed, the structure of the GR gene promoter with alternate tissue-specific untranslated first exons is conserved in rodents and humans $(64,65)$.

As in other species $(7,12,66)$, antenatal dexamethasone in the vervet produced hypertension in the juvenile offspring. Prenatal exposure to dexamethasone has previously been shown to elevate fetal and neonatal blood pressure in baboons (67-69) and in adolescent humans (30). The mechanisms of early-life programming of elevated blood pressure are unknown but, in rodent models, have been ascribed to variations in nephron number, vascular sensitivity to vasoconstrictors, and hyperinsulinemia (70-72). Urinary steroid profiling does not suggest that altered renal $11 \beta-H S D 2$ contributes to the hypertension.

Finally, the highest dose of dexamethasone also increased activation of the offspring HPA axis, at least under the stress of sampling, reflecting findings in other models of prenatal programming in a variety of species and at the diurnal maximum in low-birth-weight children and adults (refs. 27, 72; reviewed in ref. 73). Interestingly, in rhesus monkeys, stress during pregnancy also programs increased HPA activity in the offspring (74). After the World Trade Center attacks of September 11, 2001, mothers who developed posttraumatic stress disorder showed lower cortisol levels, and most interestingly, so did their babies, with the effects mostly confined to those exposed in later pregnancy (29). Mechanistically, both dexamethasone suppression (testing GR sensitivity in the anterior pituitary and, less certainly, the CNS) and basal cortisol production and metabolism were unaltered. While no test is faultless, these data suggest that the exaggerated cortisol response to stress after prenatal dexamethasone reflects increased drive rather than attenuated feedback. This contrasts with altered feedback seen in rats and other species with late-gestational dexamethasone exposure (15). In rodents, the central mechanisms of

\section{Figure 4}

Blood pressure is elevated in 12- to 14-month-old offspring of dexamethasone-treated pregnancies. Top: Blood pressure in 8-monthold offspring (no significant differences). Bottom: Blood pressure in 12- to 14-month-old offspring. Two-way repeated-measures ANOVA, interaction between blood pressure and offspring group, $\mathrm{F}_{(3,39)}=3.65$, $P<0.03$; offspring group, $\mathrm{F}_{(3,39)}=8.81, P<0.0002$; post hoc, Fisher's least significant difference method, ${ }^{*} P<0.04$.

HPA axis hyperactivity depend on the window of steroid exposure during pregnancy $(7,15)$. Human brain subregions develop at different times to analogous structures in rodents, so the differing mechanisms of dexamethasone HPA axis programming implied are not unexpected.

These data do not reveal whether the dexamethasone-programmed phenotype will persist into adult life and, if so, whether it will be exacerbated or attenuated with age; ongoing followup of the offspring may resolve this point. Data from shorter-lived models and human epidemiology suggest that the changes will diverge further from controls with aging and adult challenges such as obesity $(31,54,75)$.

In sum, in a nonhuman primate model of singleton pregnancy, antenatal exposure to low doses of dexamethasone, which had no significant effect on birth weight or other anthropometric parameters, produced disordered glucose-insulin homeostasis, reduced pancreatic $\beta$ cell mass, and elevated blood pressure and cortisol levels in juvenile offspring. The data are compatible with the extrapolation of the glucocorticoid programming hypothesis to primates.

\section{Methods}

Animals. All animals selected for this study were healthy adult vervet monkeys (Chlorocebus aethiops), also known as African green monkeys, and part of a captive-bred indoor colony at the Primate Unit of the South African Medical Research Council. Forty animals were multiparous females with an average weight of $3.35 \pm 0.42 \mathrm{~kg}$, and 13 were males with an average weight of $4.78 \pm 0.92 \mathrm{~kg}$. All were selected for normal blood pressures and glucose tolerance with the use of reference data established for this colony. The monkeys were permanently identified with numbers in ink tattoo.

Housing. For the duration of the study, all monkeys were maintained under identical housing conditions as described previously $(76,77)$. In short, the air was changed between 15 and 20 times each hour, the temperature was kept at $25^{\circ} \mathrm{C}$, the humidity was $50 \%$, and light and dark phases both lasted 12 hours. To enable mating, the animals were maintained in pairs, and then the females were housed singly during pregnancy and lactation. All individually housed animals had access to exercise cages and for-

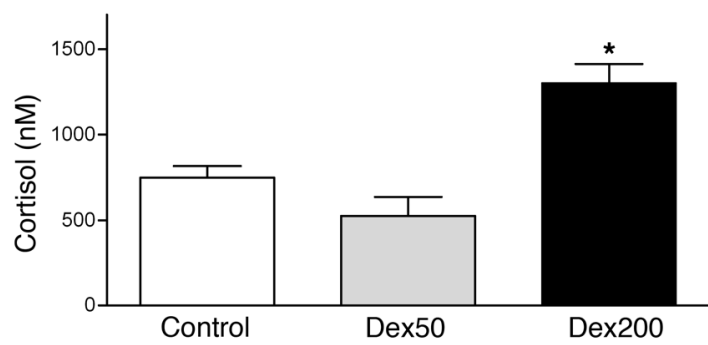

Figure 5

Plasma cortisol levels are altered in 8-month-old offspring of pregnant animals treated with dexamethasone. Cortisol levels are "stress" samples taken after brief restraint and anesthesia at $10 \mathrm{am}$. ${ }^{\star} P<0.05$ versus control, 2-tailed Student's $t$ test. 
aging material 3 times per week. Bird sounds were piped into every room. After weaning at 8 months, the offspring were kept in pairs for behavioral observations, then placed in peer groups of 6 same-sex individuals from 10 months to 12 months of age.

Pregnancy diagnosis and monitoring. Females were paired with males regardless of the stage of the menstrual cycle, and pregnancies were confirmed, dated, and monitored ultrasonographically as described by Seier et al. (78) for vervet monkeys. To diagnose pregnancy, the females were examined once a month under ketamine anesthesia at $10 \mathrm{mg} / \mathrm{kg}$ body weight. A gestational sac $2 \mathrm{~mm}$ in width is the first sign of pregnancy in vervet monkeys and indicates an embryo of about 33 days' menstrual age (78). The gestational sac dimension was measured with electronic calipers, and these measurements, as well as features such as size and date of first appearance of the yolk sac and first appearance of fetal heartbeat, were used as a basis for calculating the menstrual age of the pregnancy. This method was also used to determine a date for the start of the glucocorticoid treatment. The gestation period in vervet monkeys is about 5.5 months $(79,80)$. The gestational age was determined by previously established intrarectal digital and ultrasonographic techniques for vervet monkey pregnancies (77, 78). Pregnant vervets were monitored ultrasonically at weeks 12 and 22 to determine thickness of placenta, fetal heart rate, abdominal diameter, and femur length. Also at 12 and 22 weeks, blood samples were taken to determine anions and cortisol.

Treatments. The females were randomly divided into 4 groups, 3 of which were given dexamethasone at 50,120 , or $200 \mu \mathrm{g} / \mathrm{kg} / \mathrm{d}$ (denoted as dex 50 , dex120, and dex200) from mid-gestation onward. Dexamethasone was discontinued after birth. The dose of dexamethasone is not dissimilar to doses used in human pregnancy, which vary from $20 \mu \mathrm{g} / \mathrm{kg} / \mathrm{d}$ in congenital adrenal hyperplasia $(33,48,53)$ to $100-500 \mu \mathrm{g} / \mathrm{kg} / \mathrm{d}$ in threatened preterm labor $(30,31,35,36,45,46)$. The dexamethasone was administered daily in food, which consisted of precooked maize meal containing micro-macronutrient supplementation, as described previously (81). The diet has supported normal reproduction and development for 2 generations (76). Compliance was determined indirectly by determination of the anticipated suppression of maternal plasma cortisol by dexamethasone twice during pregnancy (as described below).

Interventions, tests, and measurements. Weight, head circumference, head length, biparietal diameter, crown-heel length, crown-rump length, abdominal circumference, subcutaneous fat thickness, femur length, tibia length, forearm length, and hip width were measured at birth with the use of tape and calipers, and at 2, 4, 6, 8, and 12 months of age. The ponderal index and Brazelton score were determined at birth. Blood was sampled from infant offspring via the femoral vein under ketamine anesthesia $(10 \mathrm{mg} / \mathrm{kg}$ body weight, intramuscularly). Ketamine was delivered under brief physical restraint in the home cage.

Adapted Brazelton score at birth. An adapted Brazelton score based on various individual measures was used as an indication of motor function at birth. These measures, followed by the score measures, included the following: (a) Palmar grasp response: place index finger in palm; 0 , no grasping reflex; 1 , partial grasp; 2 , strong reflexive grasp without voluntary release. (b) Plantar grasp response: place index finger under foot; 0 , no grasping reflex; 1, partial grasp; 2 , strong reflexive grasp without voluntary release. (c) Body righting: time noted for the infant to turn from supine to prone; 0 , partial righting; 1 , righting in 5 seconds; 2 , righting in less than 5 seconds. (d) Resistance to passive movement: degree of resistance to passive flexion and extension of limbs; 0 , barely discernible resistance; 1 , moderate resistance; 2 , strong resistance. (e) Active power: strength of muscles when actively contracting; 0 , some strength but cannot withstand slight resistance; 1 , withstands moderate resistance; 2 , extremely strong. (f) Tactile response: response to tactile stimulus to the 4 extremities (with a cotton swab);
0 , no response; 1 , barely discernible response; 2 , easily apparent response. (g) Spontaneous locomotion: quality of locomotion within 1 minute; 0 , none; 1 , a weak attempt at crawling; 2 , coordinated crawling noted. (h) Motor activity: observation of motor activity throughout the observation period.

Ponderal index. The ponderal index relates weight (in grams) to height (in $\mathrm{cm})$. The index is calculated as (weight/height $\left.{ }^{3}\right) \times 100$.

Intravenous glucose tolerance tests. Glucose tolerance was determined in the offspring at 8 months of age. Serial blood sampling was obtained via an i.v. catheter inserted into the saphenous vein under aseptic conditions and connected to a drip set containing physiological saline to maintain patency. After obtaining a baseline blood sample for glucose and insulin, $1 \mathrm{ml} / \mathrm{kg}$ of $50 \%$ dextrose was administered i.v. Further sampling for blood glucose occurred 5, 10, 15, 20, 40, and 60 minutes after dextrose. A sample for plasma insulin was taken at 10 minutes. Glucose was analyzed by an enzymatic essay (Bayer T01-1825-56) and insulin by microparticle enzyme immunoassay (AxSYM B2D010; Abbott Diagnostics).

Dexamethasone suppression test. The suppression test was conducted in the offspring at 8 and 12 months. A basal blood sampling at 10:00 pm was followed by an i.v. administration of $0.026 \mathrm{mg} / \mathrm{kg}$ dexamethasone. A second sample was obtained at 7:00 am. All samples were analyzed for cortisol as described above.

Organ weights. At 12-14 months, vervets were killed, and part of the liver and pancreas, and the whole heart, kidney, adrenal, brain, pituitary, and thymus, were removed to determine organ weight and for further examination.

PEPCK activity. At $12-14$ months, part of the liver was snap-frozen to determine PEPCK activity as described previously $(26,82)$. In short, liver tissue was homogenized and the cytosolic fraction obtained. The cytosolic fraction $(1 \mathrm{mg})$ was assayed in $1 \mathrm{ml}$ buffer containing $50 \mathrm{mM}$ HEPES ( $\mathrm{pH}$ 6.5), $50 \mathrm{mM}$ sodium bicarbonate, $1 \mathrm{mM} \mathrm{MgCl}_{2}, 0.25 \mathrm{mM} \mathrm{NADH}, 1.0$ $\mathrm{mM}$ phosphoenolpyruvate, and $1.5 \mathrm{U}$ malate dehydrogenase. The absorbance of NADH was determined at $340 \mathrm{~nm}$ at $30^{\circ} \mathrm{C}$. After a stable signal was obtained, the reaction was initiated by addition of $0.15 \mathrm{mM} 2$ '-deoxyguanosine $5^{\prime}$ diphosphate. After 3 minutes of incubation, the decrease in absorbance was measured at a wavelength of $340 \mathrm{~nm}$. The assay was performed in duplicate for all samples. A reaction mixture without bicarbonate was used as a negative control.

Real-time PCR. RNA was extracted from frozen liver aliquots with the use of TRIzol (Invitrogen). The isolated RNA was reverse-transcribed to obtain cDNA with the use of a Reverse Transcriptase kit (Reverse Transcriptase [RT] system; Promega). PEPCK and GR $\alpha$ mRNA were quantified with RT-PCR primer-probe sets with the use of the ABI PRISM 7900 Sequence Detection System (PE Applied Biosystems) with the following primers and probes: GR $\alpha$ : 5'-CATTGTCAAGAGGGAAGGAAACTC-3' (forward), 5'-GATTTTCAACCACTTCATGCATAGAA-3' (reverse), and 5'-6-FAMTTTGTCAGTTGATAAAACCGCTGCCAGTTCT-TAMRA-3' (probe). 18S ribosomal RNA primers and probes (Applied Biosystems) were used to normalize the transcript levels. A standard curve for each primer-probe set was generated in triplicate by serial dilution of cDNA that was pooled from several subjects. Each sample was run in duplicate, and the mean values of the duplicates were used to calculate the transcript level from the standard curve. The results are expressed as arbitrary units.

Pancreas histology. Pancreata were placed in 4\% paraformaldehyde overnight and processed in an automated tissue processor (Shandon Citadel 1000; Thermo Scientific) through ascending concentrations of ethanol from $70 \%-100 \%$, followed by xylene. The tissue was embedded in paraffin wax (Paraplast Plus; Monoject Scientific). Sections $4 \mu \mathrm{m}$ thick were cut on a rotary microtome and mounted on slides coated with 3-aminopropyltriethoxysilane. Serial sections were incubated for 5 minutes in $0.228 \%$ periodic acid to inhibit endogenous peroxidases. In each section, $\alpha$ cells were immunolabeled first, with a polyclonal glucagon antibody (Dako) 
for 30 minutes at room temperature followed by $0.05 \%$ diaminobenzidine tetrahydrochloride. Then $\beta$ cells were labeled with a monoclonal insulin antibody, overnight at $4{ }^{\circ} \mathrm{C}$ (Sigma-Aldrich), followed by avidinbiotin-peroxidase complex (VECTASTAIN; Vector Laboratories). Fuchsin (Dako) was used to reveal the immunolabeled insulin-secreting $\beta$ cells. In the method controls, primary antibody was omitted. All sections were counterstained with hematoxylin.

Blood pressure measurements. Blood pressure measurements were obtained with a Dinamap pediatric blood pressure machine as previously used in primates (83). Measurements were taken at 8 and 12 months of age, and the average of 5 measurements was used as a measure for blood pressure. These were obtained under ketamine anesthesia $(10 \mathrm{mg} / \mathrm{kg}$, intramuscularly); the monkeys were in a supine position with a cuff around their left upper arm.

24-Hour urine collection. Urine was collected for 24 hours by placement of a funnel covering the entire floor area of the cage. Cortisol and its metabolites were measured in urine by electron impact gas chromatography/mass spectrometry following Sep-Pak C18 extraction (Waters Corp.), hydrolysis with $\beta$-glucuronidase, and formation of the methoxime-trimethylsilyl derivatives. Epicortisol and epi-tetrahydrocortisol were used as internal standards. Total cortisol metabolite excretion was calculated as tetrahydrocortisols (THFs) + tetrahydrocortisone (THE) + cortols + cortolones. Relative metabolism by 5 - and $5 \beta$-reductases was inferred from the $5 \beta$-THF $/ 5$-THF ratio. A-ring reduction of cortisol was inferred from the ratios of THFs to cortisol and $5 \beta$-reductase activity from the ratio of THE to cortisone. Whole-body equilibrium between cortisol and cortisone, determined by the balance of tissue-specific activities of $11 \beta$-reductase and $11 \beta$-dehydrogenase, was inferred from the ratio of
THFs to THE. Renal 11ß-dehydrogenase activity was inferred from the urinary free cortisol/cortisone ratio (84).

Ethical consideration. The experiments were performed in accordance with the National Code for Animal Use in Research, Education, and Diagnosis and Testing of Drugs and Related Substances in South Africa. The study was approved by the Ethics Committee for Research on Animals of the South African Medical Research Council and by the Ethical Review committee of the University of Edinburgh.

Statistics. Data were analyzed by 1- or 2-way ANOVA with repeated measures where appropriate. Post hoc tests involved either Bonferroni's multiple-comparison test or Fisher's least significant difference method for factor as clearly indicated. Live versus still births were analyzed by $\chi^{2}$. Kruskal-Wallis nonparametric testing was used to analyze Brazelton scores, followed by Mann-Whitney $U$ testing. $P<0.05$ was regarded as significantly different.

\section{Acknowledgments}

We would like to thank Ruth Andrew and Alison Ayres for their excellent assistance with the GCMS analysis. This study was funded by a grant (to J.R. Seckl) from the Wellcome Trust.

Received for publication November 16, 2006, and accepted in revised form January 30, 2007.

Address correspondence to: Jonathan R. Seckl, Endocrinology Unit, University of Edinburgh, The Queen's Medical Research Institute, 47 Little France Crescent, Edinburgh EH16 4TJ, United Kingdom. Phone: 44-131-242-6777; Fax: 44-131-2426779; E-mail: j.seckl@ed.ac.uk.
1. Barker, D.J. 1998. In utero programming of chronic disease. Clin. Sci. 95:115-128.

2. Barker, D.J. 1999. The fetal origins of type 2 diabetes mellitus. Ann. Intern. Med. 130:322-324.

3. Hales, C.N., and Barker, D.J. 1992. Type 2 (noninsulin-dependent) diabetes mellitus: the thrifty phenotype hypothesis. Diabetologia. 35:595-601.

4. Curhan, G.C., et al. 1996. Birth weight and adult hypertension, diabetes mellitus, and obesity in US men. Circulation. 94:3246-3250.

5. Rich-Edwards, J.W., et al. 2005. Longitudinal study of birth weight and adult body mass index in predicting risk of coronary heart disease and stroke in women. BMJ. 330:1115.

6. Edwards, C.R., Benediktsson, R., Lindsay, R.S., and Seckl, J.R. 1993. Dysfunction of placental glucocorticoid barrier: link between fetal environment and adult hypertension? Lancet. 341:355-357.

7. Levitt, N.S., Lindsay, R.S., Holmes, M.C., and Seckl, J.R. 1996. Dexamethasone in the last week of pregnancy attenuates hippocampal glucocorticoid receptor gene expression and elevates blood pressure in the adult offspring in the rat. Neuroendocrinology. 64:412-418.

8. Nyirenda, M.J., Welberg, L.A., and Seckl, J.R. 2001. Programming hyperglycaemia in the rat through prenatal exposure to glucocorticoids - fetal effect or maternal influence? J. Endocrinol. 170:653-660.

9. O'Regan, D., Kenyon, C.J., Seckl, J.R., and Holmes, M.C. 2004. Glucocorticoid exposure in late gestation in the rat permanently programs gender-specific differences in adult cardiovascular and metabolic physiology. Am. J. Physiol. Endocrinol. Metab. 287:E863-E870.

10. Shen, C.N., Seckl, J.R., Slack, J.M., and Tosh, D. 2003. Glucocorticoids suppress beta-cell development and induce hepatic metaplasia in embryonic pancreas. Biochem. J. 375:41-50.

11. Welberg, L.A., Seckl, J.R., and Holmes, M.C. 2000. Inhibition of 11 beta-hydroxysteroid dehydrogenase, the foeto-placental barrier to maternal glu- cocorticoids, permanently programs amygdala GR mRNA expression and anxiety-like behavior in the offspring. Eur. J. Neurosci. 12:1047-1054.

12. Woods, L.L., and Weeks, D.A. 2005. Prenatal programming of adult blood pressure: role of maternal corticosteroids. Am. J. Physiol. Regul. Integr. Comp. Physiol. 289:R955-R962.

13. Drake, A.J., Walker, B.R., and Seckl, J.R. 2005. Intergenerational consequences of fetal programming by in utero exposure to glucocorticoids in rats. $\mathrm{Am}$. J. Physiol. Regul. Integr. Comp. Physiol. 288:R34-R38.

14. Benediktsson, R., Lindsay, R.S., Noble, J., Seckl, J.R., and Edwards, C.R. 1993. Glucocorticoid exposure in utero: new model for adult hypertension. Lancet. 341:339-341.

15. Welberg, L.A., Seckl, J.R., and Holmes, M.C. 2001 Prenatal glucocorticoid programming of brain corticosteroid receptors and corticotrophin-releasing hormone: possible implications for behavior. Newroscience. 104:71-79.

16. Chung, S., et al. 2005. Differential adaptive responses to chronic stress of maternally stressed male mice offspring. Endocrinology. 146:3202-3210.

17. Sloboda, D.M., et al. 2005. Hepatic glucose regulation and metabolism in adult sheep: effects of prenatal betamethasone. Am. J. Physiol. Endocrinol. Metab. 289:E721-E728.

18. Kanitz, E., Otten, W., Tuchscherer, M., and Manteuffel, G. 2003. Effects of prenatal stress on corticosteroid receptors and monoamine concentrations in limbic areas of suckling piglets (Sus scrofa) at different ages. J. Vet. Med. A Physiol. Pathol. Clin. Med. 50:132-139.

19. Jarvis, S., et al. 2005. Programming the offspring of the pig by prenatal social stress: neuroendocrine activity and behavior. Horm. Behav. 49:68-80.

20. Liu, L., Li, A., and Matthews, S.G. 2001. Maternal glucocorticoid treatment programs HPA regulation in adult offspring: sex-specific effects. Am. J. Physiol. Endocrinol. Metab. 280:E729-E739.

21. Brown, R.W., et al. 1996. The ontogeny of 11 beta-hydroxysteroid dehydrogenase type 2 and mineralocorticoid receptor gene expression reveal intricate control of glucocorticoid action in development. Endocrinology. 137:794-797.

22. Holmes, M.C., et al. 2006. The mother or the fetus? $11 \beta$-Hydroxysteroid dehydrogenase type 2 null mice provide evidence for direct fetal programming of behavior by endogenous glucocorticoids. J. Neurosci. 26:3840-3844.

23. Welberg, L.A., Thrivikraman, K.V., and Plotsky, P.M. 2005. Chronic maternal stress inhibits the capacity to up-regulate placental 11 beta-hydroxysteroid dehydrogenase type 2 activity. J. Endocrinol. 186:R7-R12.

24. Kapoor, A., Dunn, E., Kostaki, A., Andrews, M.H., and Matthews, S.G. 2006. Fetal programming of hypothalamo-pituitary-adrenal function: prenatal stress and glucocorticoids. J. Physiol. 572:31-44.

25. Lesage, J., et al. 2004. Prenatal stress induces intrauterine growth restriction and programs glucose intolerance and feeding behavior disturbances in the aged rat. J. Endocrinol. 181:291-296.

26. Nyirenda, M.J., Lindsay, R.S., Kenyon, C.J., Burchell, A., and Seckl, J.R. 1998. Glucocorticoid exposure in late gestation permanently programs rat hepatic phosphoenolpyruvate carboxykinase and glucocorticoid receptor expression and causes glucose intolerance in adult offspring. J. Clin. Invest. 101:2174-2181.

27. Phillips, D.I., et al. 1998. Elevated plasma cortisol concentrations: a link between low birth weight and the insulin resistance syndrome? J. Clin. Endocrinol. Metab. 83:757-760.

28. Goland, R.S., et al. 1993. Elevated levels of umbilical cord plasma corticotropin-releasing hormone in growth-retarded fetuses. J. Clin. Endocrinol. Metab. 77:1174-1179.

29. Yehuda, R., et al. 2005. Transgenerational effects of posttraumatic stress disorder in babies of mothers exposed to the World Trade Center attacks during pregnancy. J. Clin. Endocrinol. Metab. 90:4115-4118.

30. Doyle, L.W., Ford, G.W., Davis, N.M., and Calla- 
nan, C. 2000. Antenatal corticosteroid therapy and blood pressure at 14 years of age in preterm children. Clin. Sci. 98:137-142.

31. Dalziel, S.R., et al. 2005. Cardiovascular risk factors after antenatal exposure to betamethasone: 30 -year follow-up of a randomized controlled trial. Lancet. 365:1856-1862.

32. Trautman, P.D., Meyer-Bahlburg, H.F., Postelnek, J., and New, M.I. 1995. Effects of early prenatal dexamethasone on the cognitive and behavioral development of young children: results of a pilot study. Psychoneuroendocrinology. 20:439-449.

33. Spiliotis, B.E. 2001. Prenatal diagnosis and treatment of congenital adrenal hyperplasia and consequences in adults. J. Pediatr. Endocrinol. Metab. 14(Suppl. 5):1299-1302.

34. Brazelton, T.B. 1978. The Brazelton Neonatal Behavior Assessment Scale: introduction. Monogr. Soc. Res. Child Dev. 43:1-13.

35. Crowther, C.A., Haslam, R.R., Hiller, J.E., Doyle, L.W., and Robinson, J.S. 2006. Neonatal respiratory distress syndrome after repeat exposure to antenatal corticosteroids: a randomized controlled trial. Lancet. 367:1913-1919.

36. Peaceman, A.M., Bajaj, K., Kumar, P., and Grobman, W.A. 2005. The interval between a single course of antenatal steroids and delivery and its association with neonatal outcomes. Am. J. Obstet. Gynecol. 193:1165-1169.

37. Kajantie, E., et al. 2005. Size at birth as a predictor of mortality in adulthood: a follow-up of 350000 person-years. Int. J. Epidemiol. 34:655-663.

38. Novy, M.J., and Walsh, S.W. 1983. Dexamethasone and estradiol treatment in pregnant rhesus macaques: effects on gestational length, maternal plasma hormones, and fetal growth. Am. J. Obstet. Gynecol. 145:920-931.

39. Gunberg, D.L. 1957. Some effects of exogenous hydrocortisone on pregnancy in the rat. Anat. Rec. 129:133-153.

40. Fayol, L., Masson, P., Millet, V., and Simeoni, U. 2004. Cushing's syndrome in pregnancy and neonatal hypertrophic obstructive cardiomyopathy. Acta Paediatr. 93:1400-1402.

41. Costedoat-Chalumeau, N., et al. 2003. Questions about dexamethasone use for the prevention of anti-SSA related congenital heart block. Ann. Rheum. Dis. 62:1010-1012.

42. Johnson, J.W., et al. 1981. Long-term effects of betamethasone on fetal development. Am. J. Obstet. Gynecol. 141:1053-1064.

43. Schneider, M.L., Roughton, E.C., Koehler, A.J., and Lubach, G.R. 1999. Growth and development following prenatal stress exposure in primates: an examination of ontogenetic vulnerability. Child Dev. 70:263-274.

44. Lingas, R.I., and Matthews, S.G. 2001. A short period of maternal nutrient restriction in late gestation modifies pituitary-adrenal function in adult guinea pig offspring. Neuroendocrinology. 73:302-311.

45. French, N.P., Hagan, R., Evans, S.F., Mullan, A., and Newnham, J.P. 2004. Repeated antenatal corticosteroids: effects on cerebral palsy and childhood behavior. Am. J. Obstet. Gynecol. 190:588-595.

46. Bloom, S.L., Sheffield, J.S., McIntire, D.D., and Leveno, K.J. 2001. Antenatal dexamethasone and decreased birth weight. Obstet. Gynecol. 97:485-490.

47. Forest, M.G., David, M., and Morel, Y. 1993. Prenatal diagnosis and treatment of 21-hydroxylase deficiency. J. Steroid Biochem. Mol. Biol. 45:75-82.

48. Mercado, A.B., Wilson, R.C., Cheng, K.C., Wei, J.Q., and New, M.I. 1995. Prenatal treatment and diagnosis of congenital adrenal hyperplasia owing to steroid 21-hydroxylase deficiency.J. Clin. Endocrinol. Metab. 80:2014-2020.

49. de Kloet, E.R., Sibug, R.M., Helmerhorst, F.M., and
Schmidt, M. 2005. Stress, genes and the mechanism of programming the brain for later life. Neurosci. Biobehav. Rev. 29:271-281.

50. Patin, V., Vincent, A., Lordi, B., and Caston, J. 2004. Does prenatal stress affect the motoric development of rat pups? Brain Res. Dev. Brain Res. 149:85-92.

51. Rieger, M., et al. 2004. Influence of stress during pregnancy on HPA activity and neonatal behavior. Ann. N. Y. Acad. Sci. 1032:228-230.

52. Holmes, M.C., et al. 2006. 11 $\beta$-Hydroxysteroid dehydrogenase type 2 protects the neonatal cerebellum from deleterious effects of glucocorticoids. Neuroscience. 137:865-873.

53. Meyer-Bahlburg, H.F., et al. 2004. Cognitive and motor development of children with and without congenital adrenal hyperplasia after early-prenatal dexamethasone. J. Clin. Endocrinol. Metab. 89:610-614.

54. Ong, K.K. 2006. Size at birth, postnatal growth and risk of obesity. Horm. Res. 65(Suppl. 3):65-69.

55. Moss, T.J., et al. 2001. Programming effects in sheep of prenatal growth restriction and glucocorticoid exposure. Am. J. Physiol. Regul. Integr. Comp. Physiol. 281:R960-R970.

56. Fernandez-Twinn, D.S., et al. 2005. Maternal protein restriction leads to hyperinsulinemia and reduced insulin-signaling protein expression in 21-month-old female rat offspring. Am. J. Physiol. Regul. Integr. Comp. Physiol. 288:R368-R373.

57. Bergman, R.N., Finegood, D.T., and Kahn, S.E. 2002. The evolution of beta-cell dysfunction and insulin resistance in type 2 diabetes. Eur. J. Clin. Invest. 32(Suppl. 3):35-45.

58. Gesina, E., et al. 2004. Dissecting the role of glucocorticoids on pancreas development. Diabetes. 53:2322-2329.

59. Johnson, J.W., Mitzner, W., London, W.T., Palmer, A.E., and Scott, R. 1979. Betamethasone and the rhesus fetus: multisystemic effects. Am. J. Obstet. Gynecol. 133:677-684.

60. Chakravarty, K., Cassuto, H., Reshef, L., and Hanson, R.W. 2005. Factors that control the tissue-specific transcription of the gene for phosphoenolpyruvate carboxykinase-C. Crit. Rev. Biochem. Mol. Biol. 40:129-154.

61. Nyirenda, M.J., Dean, S., Lyons, V., Chapman, K.E., and Seckl, J.R. 2006. Prenatal programming of hepatocyte nuclear factor 4alpha in the rat: a key mechanism in the 'foetal origins of hyperglycaemia'? Diabetologia. 49:1412-1420.

62. Seckl, J.R., and Meaney, M.J. 2004. Glucocorticoid programming. Ann. N. Y. Acad. Sci. 1032:63-84.

63. Lillycrop, K.A., Phillips, E.S., Jackson, A.A., Hanson, M.A., and Burdge, G.C. 2005. Dietary protein restriction of pregnant rats induces and folic acid supplementation prevents epigenetic modification of hepatic gene expression in the offspring. J. Nutr. 135:1382-1386.

64. McCormick, J.A., et al. 2000. 5'-Heterogeneity of glucocorticoid receptor messenger RNA is tissue specific: differential regulation of variant transcripts by early-life events. Mol. Endocrinol. 14:506-517.

65. Turner, J.D., and Muller, C.P. 2005. Structure of the glucocorticoid receptor (NR3C1) gene 5 ' untranslated region: identification, and tissue distribution of multiple new human exon 1. J. Mol. Endocrinol. 35:283-292.

66. O'Regan, D., Welberg, L.L., Holmes, M.C., and Seckl, J.R. 2001. Glucocorticoid programming of pituitary-adrenal function: mechanisms and physiological consequences. Semin. Neonatol. 6:319-329.

67. Koenen, S.V., Mecenas, C.A., Smith, G.S., Jenkins, S., and Nathanielsz, P.W. 2002. Effects of maternal betamethasone administration on fetal and maternal blood pressure and heart rate in the baboon at 0.7 of gestation. Am. J. Obstet. Gynecol. 186:812-817.
68. Smith, L.M., Altamirano, A.K., Ervin, M.G., Seidner, S.R., and Jobe, A.H. 2004. Prenatal glucocorticoid exposure and postnatal adaptation in premature newborn baboons ventilated for six days. Am.J. Obstet. Gynecol. 191:1688-1694.

69. Ervin, M.G., Seidner, S.R., Leland, M.M., Ikegami, M., and Jobe, A.H. 1998. Direct fetal glucocorticoid treatment alters postnatal adaptation in premature newborn baboons. Am. J. Physiol. 274:R1169-R1176.

70. Woods, L.L., Weeks, D.A., and Rasch, R. 2004. Programming of adult blood pressure by maternal protein restriction: role of nephrogenesis. Kidney Int. 65:1339-1348.

71. Symonds, M.E., et al. 2003. Maternal nutrient restriction during placental growth, programming of fetal adiposity and juvenile blood pressure control. Arch. Physiol. Biochem. 111:45-52.

72. Levitt, N.S., et al. 2000. Impaired glucose tolerance and elevated blood pressure in low birth weight, nonobese, young South African adults: early programming of cortisol axis. J. Clin. Endocrinol. Metab. 85:4611-4618.

73. Matthews, S.G. 2002. Early programming of the hypothalamo-pituitary-adrenal axis. Trends Endocrinol. Metab. 13:373-380.

74. Schneider, M.L., Moore, C.F., Kraemer, G.W., Roberts, A.D., and DeJesus, O.T. 2002. The impact of prenatal stress, fetal alcohol exposure, or both on development: perspectives from a primate model. Psychoneuroendocrinology. 27:285-298.

75. Gavrilov, L.A., and Gavrilova, N.S. 2004. Early-life programming of aging and longevity: the idea of high initial damage load (the HIDL hypothesis). Ann. N. Y. Acad. Sci. 1019:496-501.

76. Lambrechts, C., Seier, J.V., and Mdhluli, C. 1999. Management and breeding of the vervet monkey (Cercopithecus aethiops) in South Africa [abstract]. In Proceedings of the international joint meeting, 12th ICLAS assembly and conference, 7th FELASA symposium. May 26-28. Mallorca, Spain. J.A. Tur-Mari et al., editors. Laboratory Animals Ltd. London, United Kingdom. 170-172.

77. Seier, J.V. 1986. Breeding vervet monkeys in a closed environment. J. Med. Primatol. 15:339-349.

78. Seier, J.V., van der Horst, G., de Kock, M., and Chwalisz, K. 2000. The detection and monitoring of early pregnancy in the vervet monkey (Cercopithecus aethiops) with the use of ultrasound and correlation with reproductive steroid hormones. J. Med. Primatol. 29:70-75.

79. Johnson, P.T., Valerio, D.A., and Thompson, G.E. 1973. Breeding the African green monkey, Cercopithecus aethiops, in a laboratory environment. Lab. Anim. Sci. 23:355-359.

80. Hess, D.L., Hendrickx, A.G., and Stabenfeldt, G.H. 1979. Reproductive and hormonal patterns in the African green monkey (Cercopithecus aethiops). J. Med. Primatol. 8:273-281.

81. Venter, F.S., Cloete, H., Seier, J.V., Faber, M., and Fincham, J.E. 1993. Folic acid and vitamin B12 status of vervet monkeys used for nutritional research. Lab. Anim. 27:59-64.

82. Petrescu, I., et al. 1979. Determination of phosphoenolpyruvate carboxykinase activity with deoxyguanosine 5'-diphosphate as nucleotide substrate. Anal. Biochem. 96:279-281.

83. Cefalu, W.T., et al. 2004. Caloric restriction and cardiovascular aging in cynomolgus monkeys (Macaca fascicularis): metabolic, physiologic, and atherosclerotic measures from a 4-year intervention trial. J. Gerontol. A Biol. Sci. Med. Sci. 59:1007-1014.

84. Andrews, R.C., Herlihy, O., Livingstone, D.E., Andrew, R., and Walker, B.R. 2002. Abnormal cortisol metabolism and tissue sensitivity to cortisol in patients with glucose intolerance. J. Clin. Endocrinol. Metab. 87:5587-5593. 